\title{
A HIGH ON/OFF RATIO MEMS CAPACITIVE SWITCH WITH APPLICATIONS IN SOLAR ENERGY HARVESTING \\ Vikram Thakar ${ }^{*}$, Zhengzheng Wu, and Mina Rais-Zadeh \\ University of Michigan, Ann Arbor, Michigan, USA
}

\begin{abstract}
In this paper, a novel electrostatic switch is reported that exhibits an ON/OFF capacitance ratio of 72 , an $\mathrm{ON}$ capacitance value of $90 \mathrm{pF}$, and a pull-in voltage of less than $5 \mathrm{~V}$ within an area of $750 \times 750 \mu \mathrm{m}^{2}$. These characteristics are achieved by utilizing a slit-shape middle metal layer in-between the top and bottom metal plates. This design makes the capacitance ratio and the ON state capacitance tolerant against fabrication and temperature induced residual stress in the suspended metal membrane, without significantly degrading the electrostatic force between the top and bottom metals.

In this three-metal configuration, at the $\mathrm{ON}$ state, the ohmic contact between the top membrane and the middle metal layer enables direct charge transfer between these two electrodes. This characteristic is attractive for device applications in solar energy harvesting. As a proof of concept, charging of an external storage capacitor is shown for an exemplary switch, when a constant current is supplied to the top electrode.
\end{abstract}

\section{INTRODUCTION}

MEMS switches are an attractive alternative to existing switch solutions and have thus been a topic of extensive research in the past few decades [1], [2]. Conventionally, an electrostatic MEMS switch is made using two electrodes separated by an air gap and a suitable dielectric. One of the electrodes is a moving membrane, and it can be pulled onto the fixed electrode with the application of an electrostatic potential. For a capacitive switch with a given device area, the up-state air gap and the down state effective dielectric thickness decide the ON and OFF capacitance, respectively. In many applications where the MEMS device is used as a switched capacitor, the switch is not only required to have a high ON/OFF capacitance ratio but also a sufficient $\mathrm{ON}$ capacitance value. Scaling up the device area to achieve a high ON capacitance is not beneficial as larger devices also exhibit an undesirably high OFF capacitance. In addition, the ON capacitance which is defined by the effective insulator thickness of the switch does not scale linearly with the device size because of warping in the movable membrane (caused by residual or thermal stress) and the surface roughness of the contacts [3]. In order to resolve these issues, a modified switch structure is presented that makes use of an additional metal layer to achieve both a high ON/OFF capacitance ratio and a high $\mathrm{ON}$ capacitance value (Fig. 1). The three-metal switch design makes the $\mathrm{ON}$ capacitance invariant to stress-induced membrane warping. A micromachined capacitive switch exhibiting an ON/OFF capacitance ratio of 72 and an ON capacitance value of $90 \mathrm{pF}$ within an area of $750 \times 750 \mu \mathrm{m}^{2}$ is demonstrated. A low pull-in voltage of less than $5 \mathrm{~V}$ is realized by designing the middle metal electrode in a slit type configuration ensuring sufficient electrostatic actuation force on the top suspended membrane. With such properties, the proposed threemetal switch design compares favorably with the state of the art MEMS switches [4].

In the ON state, the top metal makes an ohmic contact with the middle metal layer, allowing the transfer of electrostatic charge from the top membrane to the un-biased middle electrode. Such charge transfer can be useful when employing the switch as a solar energy harvester. We investigate this application and demonstrate charging of an external capacitor connected to the middle metal layer. Another unique feature of this three-metal switch when used as a harvester is the ability to deliver high-voltage charging pulses under heavy capacitive load without the need for an electrical boost converter. This is a significant advantage over conventional photovoltaic devices [5].

\section{DEVICE CONCEPT AND FABRICATION}

The three dimensional model of the proposed switch is shown in Fig. 1. The MEMS switch is actuated by applying an electrostatic potential between the top and the bottom metal layers, whereas the middle metal layer is left floating. When the top membrane pulls in, it makes an ohmic contact with the middle metal layer. Thus, the ON capacitance of the switch is mainly determined by the capacitance between the middle and bottom metal layers, and is unaffected by the curvature of the top membrane. Therefore, a repeatable and large ON capacitance can be achieved even if the top membrane is warped due to the inevitable fabrication and thermally induced residual stresses.

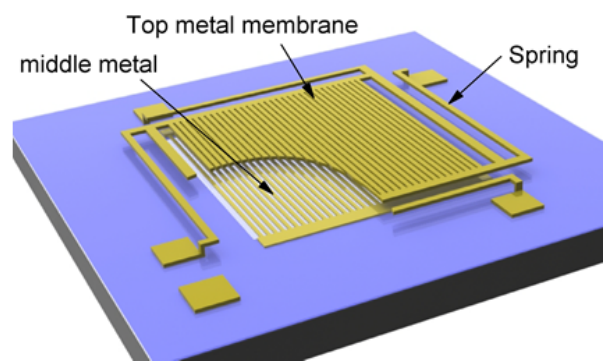

Figure 1: Schematic of the device structure with a top electrode section cut out to reveal the middle metal layer. The slit type design of the top and middle electrode can be clearly seen.

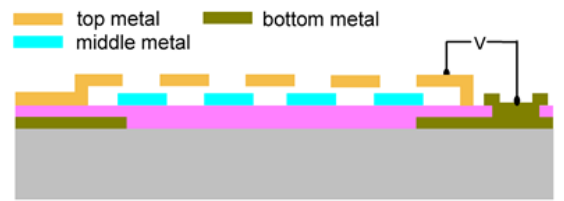

Figure 2: Cross-section of the proposed switch structure. The floating middle metal layer can be used as an additional terminal. Note that in this configuration, the substrate acts as the ground electrode.

To minimally affect the electric field distribution between the top and bottom metal layers, the metal layers are designed in a slit configuration (Fig. 2) and the middle metal layer is shifted laterally (Fig. 3). From the electrostatic simulations shown in Fig. 3, the slit-shaped middle metal layer is seen to have no significant effect on the electric field between the top and bottom metal layers. As a result, the electrostatic force on the suspended top metal remains unaffected. This allows us to design the membrane with a sufficient restoring force to overcome stiction, while still actuating the device with relatively small voltages.

The fabrication process flow for the proposed devices is depicted in Fig. 4. Starting with a nominal silicon wafer, a $500 \mathrm{~nm}$ 
thick aluminum $(\mathrm{Al})$ / chrome $(\mathrm{Cr})$ layer is lift-off patterned as the first metal layer (or ground electrode). A $150 \mathrm{~nm}$ thick aluminum oxide $\left(\mathrm{Al}_{2} \mathrm{O}_{3}\right)$ layer is deposited using atomic layer deposition (ALD) as the dielectric layer. This material and its thickness along with the metal area define the ON state capacitance of the device. The dielectric layer is patterned to enable contact to the bottom metal layer. A $100 \mathrm{~nm}$ thick gold is evaporated and lift-off patterned as the middle floating electrode with $\mathrm{Cr}$ as the adhesion layer. Poly-methyl-methacrylate (PMMA) is spun as the sacrificial layer with a thickness of $1.7 \mu \mathrm{m}$ which defines the air gap between the top and the floating electrode. Using titanium (Ti) as the etch mask, PMMA is patterned using a low power $\mathrm{O}_{2}$ plasma. Post PMMA etching, the Ti mask is removed and a 10/900 $\AA$ $\mathrm{TiW} /$ silver layer is sputtered as a seed layer for electroplating. The plating mold is created using AZ 9260 photoresist and an $8 \mu \mathrm{m}$ thick top electrode is subsequently electroplated. In the final step, the devices are released by removing the PMMA sacrificial layer and dried using critical point drying (CPD) to prevent stiction. The SEM images of a fabricated device are shown in Fig. 5.

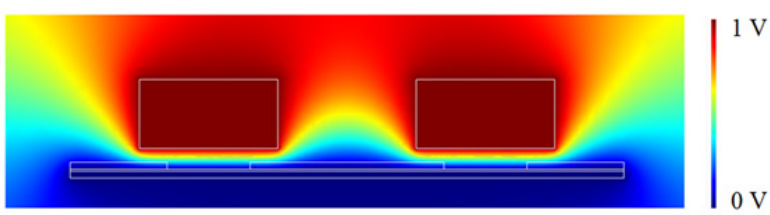

(a)

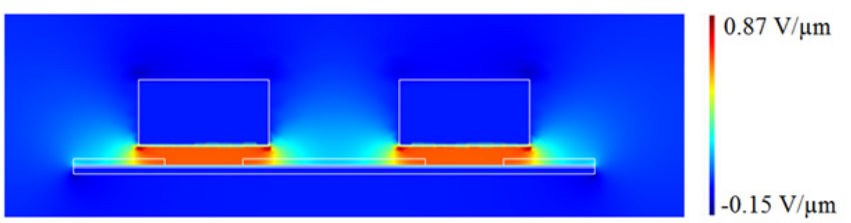

(b)

Figure 3: Simulated (a) electric potential and (b) electric field distribution of the proposed electrode layout for a two finger section. From (b) a uniform electric field is seen in between the top and bottom electrodes. The electric field is not disturbed significantly due to the addition of the middle metal layer. The two scale bars plot the range of voltage and electric field.
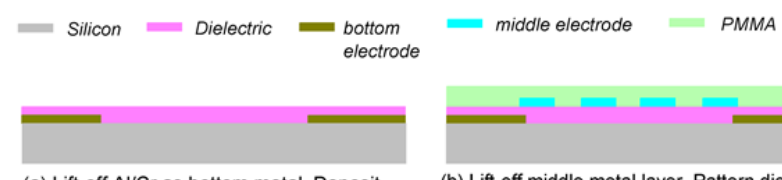

(a) Lift-off $\mathrm{Al} / \mathrm{Cr}$ as bottom metal. Deposit

$150 \mathrm{~nm} \mathrm{Al} \mathrm{O}_{3}$ as dielectric layer.

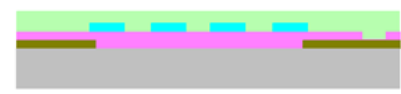

(b) Lift-off middle metal layer. Pattern dielectric to create contact to bottom metal. Spin PMMA as sacrificial layer

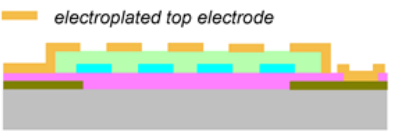

(c) Sputter plating seed layer. Electroplate top metal layer.

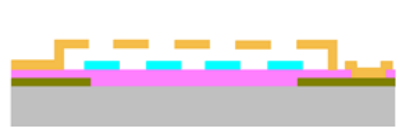

(d) Etch plating seed layer and release structure by removing sacrificial layer.

Figure 4: Process flow of the proposed MEMS capacitive switch.

\section{SWITCH DEVICE DESIGN}

There are two primary design objectives in released membrane type electrostatic switches: low pull-in voltage and large restoring force. Both can be controlled by optimizing the stiffness of the springs, and the membrane shape and area. Using the process shown in Fig. 4, the membrane and the spring

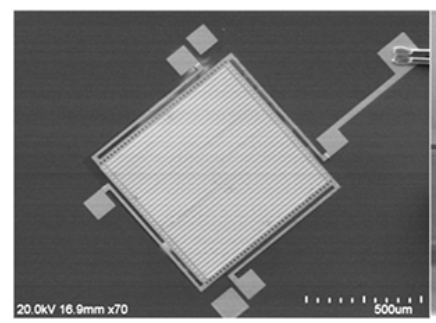

(a)

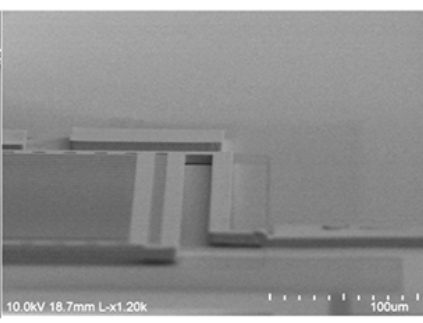

(b)
Figure 5: (a) Top SEM view of a micro-fabricated MEMS switch and (b) incline view of the top membrane revealing the air gap.

thickness is constrained to be the same to reduce the fabrication complexity. The spring stiffness is varied using lithographically defined dimensions, as shown in Fig. 6. A crab leg type spring design is implemented to allow self-compensation against fabrication induced in-plane stresses. While such a design is robust against in-plane stresses, the membrane is prone to bending under a vertical stress gradient commonly observed in most electroplated metals [6]. Figure 7 plots the simulated and measured top membrane bow due to a stress gradient in the top electroplated gold layer. Using a close to optimum plating condition, a membrane bow of $\sim 2 \mu \mathrm{m}$ was measured. From finite element simulations, a stress gradient of $\sim 10 \mathrm{MPa} / \mu \mathrm{m}$ in the top membrane was found to fit the measured bow. Using other plating conditions, higher stress gradients have been measured. With such values of inevitable stress in the top membrane, designing a traditional MEMS capacitive switch with large capacitance values is challenging [7]. In a typical two electrode switch, the membrane bow necessitates application of a voltage higher than pull-in to flatten the membrane and achieve a full area contact [8]. In the proposed design, the capacitance between the middle metal and the bottom metal layer is set by the layout of the two electrodes and the dielectric material thickness in between. As such, a high ON state capacitance is obtained even when the top membrane is only partially in contact with the middle metal layer. Therefore, although warping of the top membrane makes a uniform contact difficult, the ON capacitance value remains unaffected. This is clearly seen from the measurement results shown in Fig. 8.
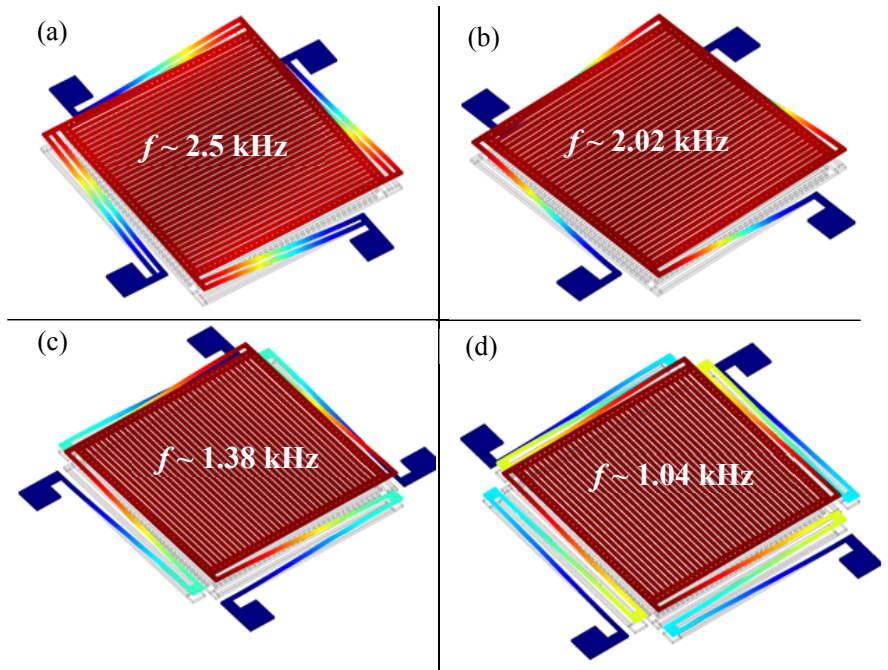

Figure 6: Modal simulation results for (a) double beam (b) single beam (c) single fold beam and (d) double fold beam designs. The color spectrum plots the total displacement. The frequency shown is a measure of maximum switching speed. 

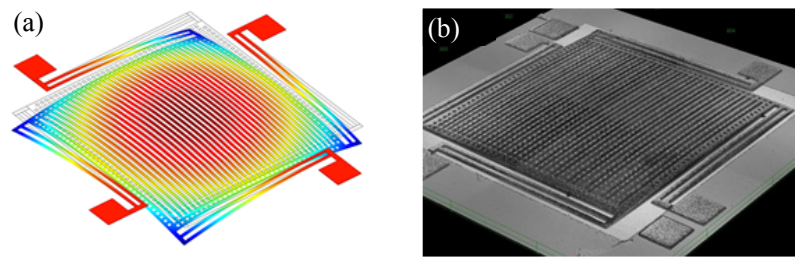

Figure 7: (a) Simulated 2 um top metal membrane bow with a stress gradient of $10 \mathrm{MPa} / \mu \mathrm{m}$ (b) Actual membrane bow measured using optical interferometry. The measured bow is $\sim 2 \mu \mathrm{m}$, indicating the stress gradient in gold is $\sim 10 \mathrm{MPa} / \mu \mathrm{m}$.

\section{SWITCH MEASUREMENTS}

A Keithley 4200 semiconductor characterization system is used to measure the capacitance vs voltage (CV) curves for the fabricated devices. Figure 8 plots the measured $\mathrm{CV}$ curves for a sample device of each spring design. As expected, the pull-in voltage is seen to reduce as the spring compliance is increased.
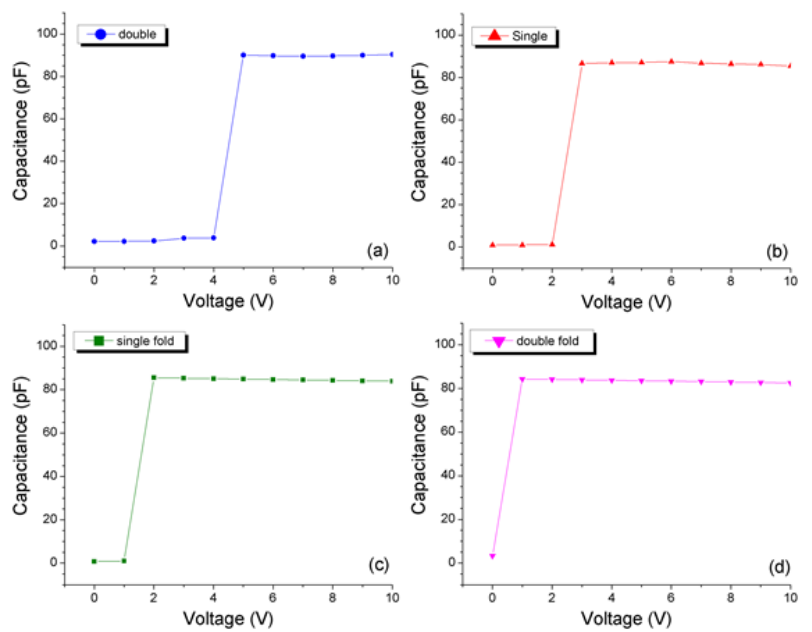

Figure 8: Measured CV curves for the (a) double beam, (b) single beam, (c) single fold beam, and (d) double folded beam spring designs. $C_{O N}$ is similar for all 4 designs. The capacitance of the measurement pads is de-embedded from the measurements.

In order to measure the switching time, DC voltage pulses are applied to the top electrode using an Agilent 81150A signal generator. The output voltage is read from the middle floating metal using an Agilent MSO7104A oscilloscope. The experimental setup is shown in Fig. 9 and the measured results are plotted in Fig. 10. When the input DC voltage is greater than the pull-in voltage, the top metal is pulled onto the middle metal and the output voltage follows the input voltage. Once the input voltage falls below the pull-in potential, the top membrane is pulled away, contact between the top and middle metal layers is broken, and the output voltage goes back to $0 \mathrm{~V}$. From Fig. 10(b), a switching time of $\sim 120 \mu$ s is measured. The results are summarized in Table 1 .

Table 1: Measured switch parameters. The switching time is estimated using equations provided in [1] assuming a mechanical $Q$ of 1.5 and applied voltage $=1.4 \times$ pull-in voltage.

\begin{tabular}{|c|c|c|c|c|c|}
\hline \multirow{2}{*}{$\begin{array}{c}\text { Spring } \\
\text { type }\end{array}$} & \multirow{2}{*}{$\begin{array}{l}\mathrm{C}_{\mathrm{ON}} \\
(\mathrm{pF})\end{array}$} & \multirow{2}{*}{$\mathrm{C}_{\mathrm{ON}} / \mathrm{C}_{\mathrm{OFF}}$} & \multirow{2}{*}{$\begin{array}{c}\text { Pull-in } \\
\text { voltage (V) }\end{array}$} & \multicolumn{2}{|c|}{ Switching time $(\mu \mathrm{s})$} \\
\hline & & & & Modeled & Measured \\
\hline Double & 90.69 & 72.7 & $<5$ & $48-146$ & - \\
\hline Single & 87.48 & 71.7 & $<3$ & $60-180$ & - \\
\hline Single fold & 85.5 & 65.3 & $<2$ & $88-264$ & 120 \\
\hline Double fold & 84.33 & 29.4 & $<1$ & $117-350$ & - \\
\hline
\end{tabular}

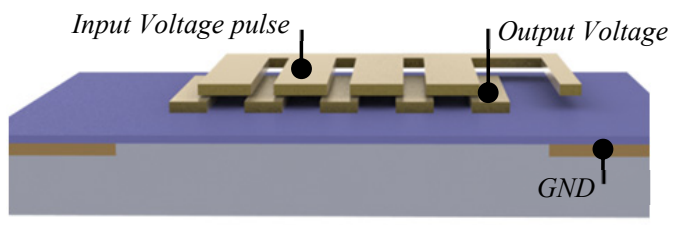

Figure 9: Experimental setup to measure the electrostatic switch response. A DC voltage pulse of $1.2 \mathrm{~V}$ is applied to the top metal membrane. The output is monitored from the middle floating layer using an oscilloscope.
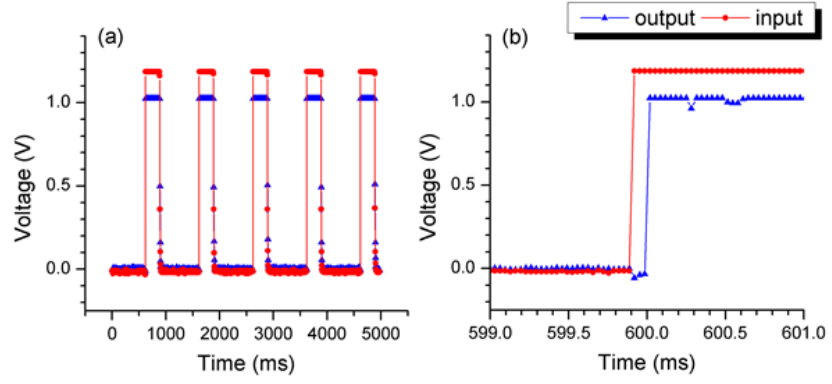

Figure 10: A voltage pulse $\left(V>V_{\text {pull-in }}\right)$ is applied to the top electrode of a single folded beam device to study the pull-in behavior of the membrane. The blue curve shows the detected output from the middle metal electrode. (a) Repeatable operation for multiple cycles and (b) delay of $\sim 120 \mu \mathrm{s}$ is measured between the input and output pulses, indicting the switching time.

While, extensive repeatability testing has not been performed, direct metal to metal contact during pull-in can cause stiction - a typical failure mechanism in ohmic switches. In such cases, the device reliability may be improved by increasing the restoring force but at the cost of increased pull-in voltage. The slit metal layer design and a large device area help reduce the actuation voltage even when the spring stiffness is large. In addition, hard contact metals, such as ruthenium [9], can be sputtered to improve the switch reliability. During high-power operations, large $\mathrm{AC}$ signal excitation induces an effective AC driving force on the switch membrane and causes self-actuation [1]. In the proposed switch design, the effective AC force is the electrostatic force between the top and bottom metal layers. Designing a switch with a larger pull-in voltage can make the device more tolerant to $\mathrm{AC}$ self-actuation and can improve the power handling.

\section{ENERGY HARVESTER APPLICATION}

The introduction of a third metal layer in the switch, as presented, opens up novel applications such as incident solar energy harvesting. The three-metal layer switch can be used as a solar energy harvester by coating the top membrane with a photosensitive material [5]. Figure 11 shows the conceptual schematic describing the solar energy harvester architecture.

During operation, the top metal layer accumulates charges due to incident radiation, which in turn causes an increase in the top membrane potential. Once pull-in voltage is reached, the top and middle metal layers will be shorted, and the accumulated charge is transferred to an external capacitor or a rechargeable battery connected to the middle metal layer, as shown in Fig. 11. Once the top metal loses all its charge, its potential drops and the membrane is lifted back up. Such electrostatic charging and discharging cycles will continue as long as energy is incident on the photosensitive material. As the maximum voltage on the top membrane is dependent on the pull-in voltage, high voltage pulses can be directly generated via design of higher pull-in voltage devices. Therefore, the proposed switch behaves as an 
electromechanical DC booster, which overcomes the heavy loading of an ultra-capacitor and improves the electrostatic charge transfer, efficiency. To experimentally verify the application of the device as a solar energy harvester, the photocurrent is replaced by a current source to inject charge on the top metal membrane. The test setup is shown in Fig. 12. In each pull-in cycle, charge is transferred to an external $1 \mu \mathrm{F}$ capacitor. The output voltage across the external capacitor is monitored using an oscilloscope with terminal impedance of $1 \mathrm{M} \Omega$. The measured voltage across the external capacitor when a constant current is supplied to the top electrode is shown in Fig. 13.

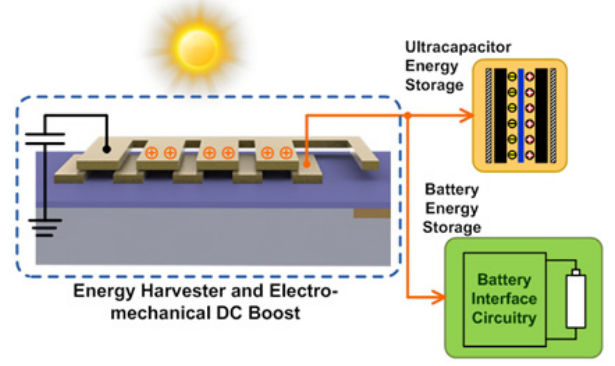

Figure 11: The proposed device architecture as envisioned for solar energy harvesting application. The top electrode should be coated with a photo-sensitive material.

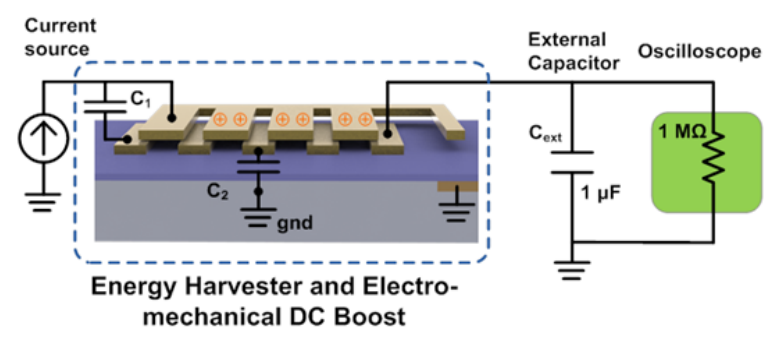

Figure 12: Experimental test setup for measuring the device performance as an energy harvester. A $1 \mu F$ external capacitor is used for collecting charge and the voltage across the capacitor is measured using an oscilloscope (with an impedance of $1 \mathrm{M \Omega}$ ).
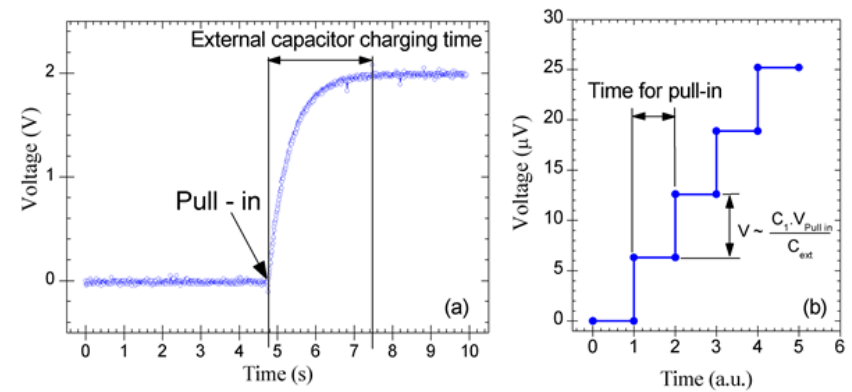

Figure 13: (a) Measured voltage across the external capacitor when a current of $2 \mu \mathrm{A}$ is connected to the membrane. As the current source is switched on, the potential on the top membrane increases, causing the membrane to pull-in and the $1 \mu F$ capacitor to charge. The output voltage is limited by the oscilloscope input impedance. (b) Theoretical plot for the voltage across the external capacitor for the first few cycles. The time step corresponds to the time required to achieve pull-in due to the photocurrent.

Using simple charge balance, the harvester performance can be analyzed. The equations for output voltage, charge collected, and efficiency of charge transfer can be written as
$V_{\text {output }}=\left(\frac{C_{1}}{C_{2}+C_{\text {ext }}}\right) V_{\text {pull in }}$

$Q_{\text {output }}=\left[\frac{C_{1} C_{\text {ext }}}{C_{2}+C_{\text {ext }}}\right] V_{\text {pull in }}$

$\eta_{\text {charge transfer }}=\left(\frac{C_{\text {ext }}}{C_{2}+C_{\text {ext }}}\right)$,

where $C_{1}, C_{2}$, and $C_{\text {ext }}$ are the capacitances in the structure as marked in Fig. 12. Using above equations, the switch design can be optimized for efficient harvester performance. From equations (1) and (2) we can see that the charge collected per cycle and the output voltage can be increased by increasing the pull-in voltage of the switch, and can thus be controlled via the spring design. This is a significant advantage over conventional photovoltaic devices, which suffer from low output voltage levels [10].

\section{CONCLUSION}

We proposed and verified a novel electrostatic switch design with an additional metal layer, which offers an improved ON/OFF capacitance ratio robust against fabrication induced residual stress. As a proof of concept, a switch with a capacitance ratio of 72 and a pull-in voltage of less than $5 \mathrm{~V}$ was demonstrated. We also investigated the use of a switch for energy harvesting applications and demonstrated feasibility of the concept though charging of an external capacitor. With their minimal dead area and the ability to generate high voltage pulses, arrays of such structures may be successfully implemented to power wireless sensor nodes.

\section{ACKNOWLEDGEMENT}

The authors thank Yonghyun Shim and the staff at the Lurie Nanofabrication facility (LNF) for their assistance. This work was supported by NSF under award \# 1055308.

\section{REFERENCES}

[1] G. M. Rebeiz, RF MEMS Theory, Design, and Technology, New York: Wiley, chapter 5, pp. 121-156, 2003.

[2] G. M. Rebeiz and J. B. Muldavin, "RF MEMS switches and switch circuits," IEEE Microwave Magazine, Dec. 2001.

[3] D. Peroulis et al., "Electromechanical considerations in developing low-voltage RF MEMS switches," IEEE Trans. Microwave Theory Tech., vol. 51, pp. 259-270, Jan. 2003.

[4] R. Al-Dahleh; R. Mansour, "High-capacitance-ratio warpedbeam capacitive MEMS switch designs," JMEMS, vol.19, no.3, pp.538-547, June 2010.

[5] Y. Huang et al., "Energy harvesting using RF MEMS," Electronic Components and Technology Conference, 2010.

[6] Y. Shim, Z. Wu, and M. Rais-Zadeh, "A multi-metal surface micromachining process for tunable RF MEMS passives," IEEE J. of Microelectromechanical Systems, 2012.

[7] A. Chinthakindi et al., J. Electrochem. Soc., vol. 149, issue 8, pp. H139-H145, 2002.

[8] Y. Shim, Z. Wu, and M. Rais-Zadeh, "A high-performance temperature-stable continuously tuned MEMS capacitor," IEEE MEMS, Cancun, Mexico, Jan. 2011, pp. 752-755.

[9] C. D. Patel and G. M. Rebeiz, "An RF-MEMS switch with $\mathrm{mN}$ contact forces," IEEE Int. Microwave Symp., May 2010.

[10] J. Christmann et al, "Bringing robustness and power efficiency to autonomous energy harvesting microsystems," Symp. on Asynchronous Circuits and Systems, 2010.

\section{CONTACT}

*Vikram Thakar, tel: +1-734-355-3480; thakar@umich.edu 